\title{
Social cognitive predictors of well-being in African college students ${ }^{2 / 3}$
}

\author{
Robert W. Lent ${ }^{\mathrm{a}, *}$, Maria do Céu Taveira ${ }^{\mathrm{b}}$, Joana Carneiro Pinto ${ }^{\mathrm{b}}$, Ana Daniela Silva ${ }^{\mathrm{b}}$, \\ Ángeles Blanco ${ }^{c}$, Susana Faria ${ }^{\mathrm{d}}$, Arminda Manuela Gonçalves ${ }^{\mathrm{d}}$ \\ a Department of Counseling, Higher Education, and Special Education, University of Maryland, College Park, MD 20742, USA \\ ${ }^{\mathrm{b}}$ Department of Applied Psychology, University of Minho, Braga, Portugal \\ c Department of Educational Research Methodology, Complutense University of Madrid, Spain \\ ${ }^{\mathrm{d}}$ Department of Mathematics, University of Minho, Braga, Portugal
}

\section{A R T I C L E I N F O}

\section{Article history:}

Received 18 January 2014

Available online 3 February 2014

\section{Keywords:}

Social cognitive career theory

Self-efficacy

Academic satisfaction

Life satisfaction

Positive affect

Well-being

\begin{abstract}
A B S T R A C T
Lent and Brown's $(2006,2008)$ social cognitive model of work well-being was tested in two samples of African college students, one from Angola $(N=241)$ and one from Mozambique $(N=425)$. Participants completed domain-specific measures of academic self-efficacy, environmental support, goal progress, and satisfaction, along with measures of global positive affect and life satisfaction. Path analyses indicated that the model fit the data well overall, both in the full sample and in separate sub-samples by country and gender. Contrary to expectations, however, self-efficacy predicted academic satisfaction only indirectly, via goal progress; and goal progress predicted life satisfaction only indirectly, via academic satisfaction. The predictors accounted for substantial portions of the variance in both academic domain satisfaction and life satisfaction. Implications for research and practice involving the social cognitive model are considered.
\end{abstract}

(c) 2014 Elsevier Inc. All rights reserved.

\section{Introduction}

The social cognitive model of educational and vocational well-being (Lent \& Brown, 2006, 2008) represents an effort to extend the explanatory range of social cognitive career theory (SCCT; Lent, Brown, \& Hackett, 1994). SCCT originally consisted of models of educational and vocational interest, choice, and performance. The newer well-being model seeks to explain satisfaction and other aspects of positive adjustment to school and work environments. Like the earlier SCCT models, the well-being model draws on basic elements of general social cognitive theory (Bandura, 1997), yet it also incorporates a variety of additional (e.g., affective, personality) variables from the literatures on domain (e.g., job) and overall life satisfaction.

A general version of the well-being model (Lent, 2004) is shown in Fig. 1. The model posits that people are more likely to be satisfied with particular life domains, such as school and work, to the extent that they (a) engage in goal-directed activity and make progress at their central goals in that domain; (b) perceive themselves as efficacious at and (c) as likely to receive positive outcomes through their involvement in the activities of that domain (i.e., high self-efficacy and outcome expectations); and (d) have access to environmental supports and resources that enable them to successfully pursue their goals or otherwise to perform effectively in that domain. In addition, domain satisfaction is seen as partly a reflection of (e) certain personality traits (e.g., one's characteristic level of positive affect) and (f) level of overall life satisfaction (i.e., a general tendency to be happy across life domains). The model also posits that life satisfaction is likely to result from the possession of favorable personality traits, progress in the pursuit of one's key domain-specific goals, and satisfaction in one's central life domains (i.e., the relation between domain and overall life satisfaction is seen as bidirectional).

\footnotetext{
is An earlier version of this study was presented at the annual meeting of the American Psychological Association, Orlando, FL, August, 2012.

* Corresponding author.

E-mail address: boblent@umd.edu (R.W. Lent).
} 


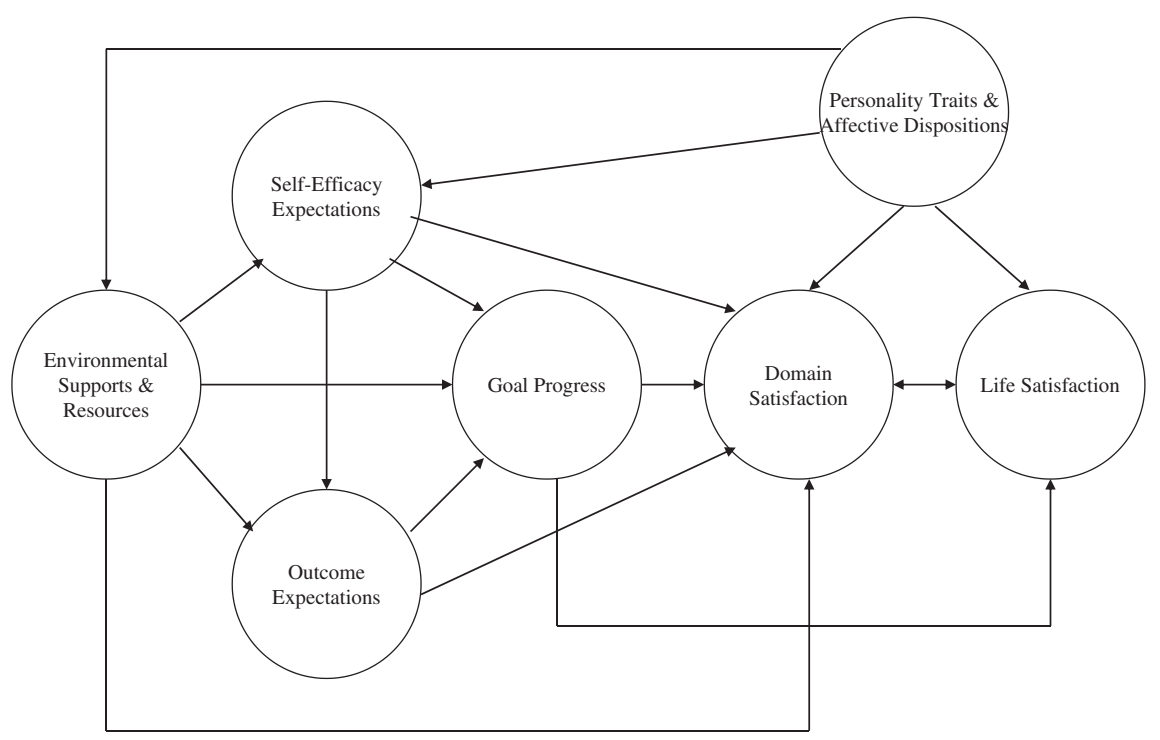

Fig. 1. Social cognitive model of domain and life satisfaction. From Lent (2004). Reprinted with permission.

In addition to these direct paths to domain and life satisfaction, the model specifies a number of linkages among the predictors of domain and life satisfaction. For example, people are more likely to make progress at their goals to the extent that they hold favorable self-efficacy and outcome expectations and experience ample environmental supports relative to their goal pursuit. Self-efficacy and environmental support facilitate goal progress both directly and indirectly by promoting positive outcome expectations. Likewise, particular personality traits, such as general positive affect, nurture goal progress and domain satisfaction indirectly by promoting positive perceptions of self-efficacy and of the availability of environmental support.

In a cross-sectional test of the well-being model in general samples of U.S. college students, Lent et al. (2005) found good fit to the data in predicting both academic and social domain satisfaction. Other cross-sectional studies have found that full or abbreviated versions of the model also offered good overall fit to the data in predicting academic satisfaction in college samples comprised of Mexican American students (Ojeda, Flores, \& Navarro, 2011), Asian American students (Hui, Lent, \& Miller, 2013), and (predominantly) European American engineering students (Lent, Singley, Sheu, Schmidt, \& Schmidt, 2007). The Ojeda et al. and Hui et al. studies each added culture-specific variables to the social cognitive model, finding that acculturation and/or enculturation were linked to academic satisfaction indirectly through various social cognitive predictors. Feldt (2012) reported

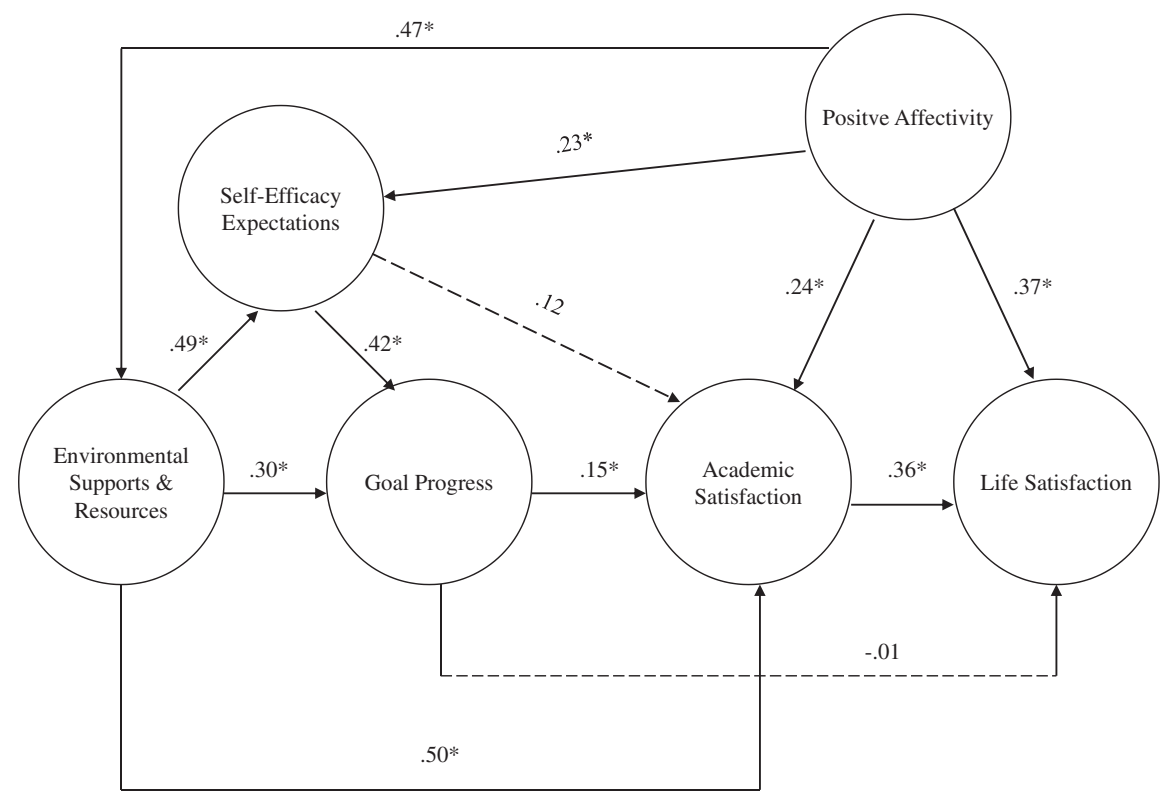

Fig. 2. Structural coefficients from the test of the abbreviated model of academic and life satisfaction in the full sample $(N=666){ }^{*} p<.05$. 
that a version of the social cognitive model usefully predicted college students' satisfaction with their career development and their college's institutional resources.

Although the well-being model has mostly been studied cross-sectionally in U.S. college samples to this point, there have been a few studies involving international college samples, adult workers, and/or longitudinal designs. For example, Singley, Lent, and Sheu (2010) generally found support for the model in a longitudinal study with American college students. Cross-sectional (Lent, Taveira, \& Lobo, 2012, Study 1) and longitudinal (Lent et al., 2012, Study 2; Lent, Taveira, Sheu, \& Singley, 2009) studies have found support for a version of the model predicting academic adjustment (e.g., satisfaction, stress) in Portuguese college students. Cross-sectional studies in the U.S. (Duffy \& Lent, 2009), Italy (Lent et al., 2011), and the United Arab Emirates (Badri, Mohaidat, Ferrandino, \& Mourad, 2013) found that the model helped account for school teachers' job satisfaction. In addition to academic or job satisfaction, several studies have found that the social cognitive model predicted overall life satisfaction (e.g., Feldt, 2012; Lent et al., 2005, 2011, 2012; Ojeda et al., 2011; Singley et al., 2010). Finally, studying adult career clients in Belgium, Verbruggen and Sels (2010) reported that the model predicted career satisfaction six months after counseling.

The present study was designed to replicate and extend existing research on the well-being model in several ways. First, although the model has been studied in the U.S. and Europe (Italy, Portugal, and Belgium), only one study has thus far examined it in a non-Western cultural context (Badri et al., 2013). Thus, there remains a need to consider the breadth of the model's cross-cultural utility. Second, there has been relatively little research comparing the model's explanatory value among women versus men (Ojeda et al., 2011). Given these gaps in research on the model, the goals of the present study were two-fold: (a) to test the well-being model in two African countries, Angola and Mozambique; and (b) to examine adequacy of model fit across countries and gender. We employed a cross-sectional design to examine the concurrent relations among the theoretical variables in an abbreviated version of the model (see Fig. 2). In particular, consistent with some other research on the well-being model (e.g., Hui et al., 2013), we did not include outcome expectations because support for the paths from this variable to goal progress and domain satisfaction has been inconsistent in prior studies (e.g., Lent et al., 2005; Ojeda et al., 2011).

\section{Method}

\subsection{Participants}

Participants were 666 students (250 women, 416 men) enrolled in undergraduate studies at universities in Angola $(N=241)$ and Mozambique $(N=425)$. Their mean age was 27.65 years, $S D=8.13$. Most of the students $(75 \%)$ were in their first or second year of college. Majors included education (72\%), management (16\%), geography (9\%), and international relations (3\%).

\subsection{Procedure and instruments}

Students were recruited for participation within intact classes. Data were gathered near the end of an academic semester so that first-year students would have some prior college experience as a basis for their responses to the assessment. After completing an informed consent form, participants were administered a Portuguese version of the survey (Angola and Mozambique are Portuguese-speaking countries) in their classrooms. The survey included measures of academic self-efficacy, goal progress, environmental support, and satisfaction; life satisfaction and trait positive affect; and demographic and academic status. The Portuguese versions of each scale were previously employed by Lent et al. (2009, 2012). Students did not receive incentives to participate in the study.

For each scale, total scores were obtained by summing item responses and dividing by the number of items on the scale. Higher scores on all scales reflected more positive expectations or experiences (e.g., stronger self-efficacy, greater satisfaction). Coefficient alpha estimates for each scale in the current sample are shown in Table 1; the values ranged from .70 to .83. Earlier research has found satisfactory reliability (e.g., coefficient alpha values of .76 to .88) and validity estimates using the Portuguese version of these measures (e.g., theory-consistent correlations with other variables in the model; Lent et al., 2009, 2012).

Self-efficacy was assessed with an 11-item scale asking students to indicate their confidence in their ability to perform well academically and to cope with barriers to academic success (e.g., "deal with lack of support by the teachers or supervisors"). Responses were obtained along a 10-point scale, ranging from no confidence ( 0 ) to complete confidence (9). The goal progress

Table 1

Means, standard deviations, correlations, and internal consistency estimates.

\begin{tabular}{|c|c|c|c|c|c|c|c|c|c|}
\hline Variable & 1 & 2 & 3 & 4 & 5 & 6 & $M$ & $S D$ & $\alpha$ \\
\hline 1. Self-efficacy & - & & & & & & 7.18 & 1.04 & .78 \\
\hline 2. Goal progress & .45 & - & & & & & 4.10 & .51 & .83 \\
\hline 3. Support & .41 & .42 & - & & & & 4.11 & .47 & .70 \\
\hline 4. Acad. satisfac. & .42 & .47 & .55 & - & & & 4.16 & .46 & .71 \\
\hline 5. Positive affect & .31 & .35 & .34 & .45 & - & & 3.76 & .54 & .78 \\
\hline 6. Life satisfac. & .24 & .25 & .31 & .38 & .40 & - & 4.62 & 1.08 & .70 \\
\hline
\end{tabular}

Note. $N=666$. Acad. satisfac. $=$ Academic satisfaction; Life satisfac. $=$ Life satisfaction. All correlations significant, $p<.001$. 
measure asked students to indicate the amount of progress they were making at eight academic goals (e.g., "actively participate in class") along a scale from 1 (no progress) to 5 (excellent progress).

Environmental support was assessed with nine items reflecting conditions that may support students' academic progress (e.g., "I am encouraged by my friends to go on with my studies".) Participants responded by indicating their level of agreement with each statement, from 1 (strongly disagree) to 5 (strongly agree). The measure of academic domain satisfaction included seven items asking students to indicate their satisfaction with their academic life (e.g., "In general, I am satisfied with my academic life") along a 1 (strongly disagree) to 5 (strongly agree) scale.

The 10-item Positive Affect (PA) scale of the Positive and Negative Affect Schedule (Watson, Clark, \& Tellegen, 1988) was used to assess the tendency to experience positive emotions. Participants responded by indicating the extent to which they generally feel each positive emotion (e.g., "proud"), from 1 (very little or not at all) to 5 (extremely). Life satisfaction was measured with the Satisfaction With Life Scale (SWLS; Diener, Emmons, Larsen, \& Griffin, 1985), which asks participants to rate their level of agreement ( 1 = strongly disagree; 7 = strongly agree) with five statements, such as "I am satisfied with my life".

\section{Results}

Table 1 presents the means, standard deviations, intercorrelations, and internal consistency reliability estimates of the measured variables in the full sample $(N=666)$. To explore possible mean differences as a function of country and gender, we ran a 2 (Angola vs. Mozambique) $\times 2$ (women vs. men) multivariate analysis of variance. This analysis yielded significant $(p<.01)$ main effects for country $\left(F[6,657]=7.67\right.$, Wilks' lambda $\left.=.94 ; \eta^{2}=.06\right)$ and gender $(F(6,657)=3.78$, Wilks' lambda $\left.=.97 ; \eta^{2}=.03\right)$. The two-way interaction was, however, non- significant. Follow-up univariate analyses of variance on the main effects revealed that the effect sizes of the statistically significant mean differences were uniformly small (none of the $\eta^{2}$ values exceeded .01). We, therefore, combined the data over country and gender in the full sample analyses.

A series of structural equation modeling analyses were next conduced to test the well-being model. These analyses employed multiple observed indicators of each latent construct to control for measurement error. Self-efficacy was represented by two indicators (academic milestone and coping self-efficacy scale scores), and item parcels were used to create multiple indicators for each of the other constructs (Bentler \& Wu, 2005). Goal progress, support, academic satisfaction, and positive affect were each represented by three item parcel scores, with 2-4 items per parcel. Life satisfaction was indexed by two parcels of 2-3 items apiece. Variance/covariance matrices (which can be obtained from the first author) were used as the input data for each model test.

Model-data fit was assessed with the comparative fit index (CFI), the root mean squared error of approximation (RMSEA), and the standardized root mean squared residual (SRMR). According to Hu and Bentler (1999), good model-data fit may be inferred from SRMR values close to .08 in combination with CFI values close to .95 or RMSEA values close to .06. Because of multivariate non-normality (Mardia's normalized estimate $>5$ ), robust maximum likelihood estimation was employed in calculating the CFI and RMSEA indices.

We first examined the tenability of a measurement model representing the six constructs in the well-being model as separate but correlated latent dimensions. In testing the measurement model, one factor loading for each construct was fixed to 1 ; all other loadings and paths among the latent constructs were freely estimated. Structural model tests were then run to assess the extent to which the hypothesized paths among the constructs were consistent with the model shown in Fig. 2 . We used two indices to compare the fit of alternative models: the scaled difference in Satorra-Bentler (S-B) $\chi^{2}$ statistic (Satorra \& Bentler, 2001) and the difference in CFI values between models. The $\Delta S-B \chi^{2}$ tests for statistically significant differences between nested models. However, because it is affected by sample size and model complexity, we also examined the $\Delta$ CFI statistic as an indicator of the practical significance of differences in model fit, using CFI differences $>.01$ as the basis for determining whether two models differ to a meaningful degree (Cheung \& Rensvold, 2002).

\subsection{Full sample analysis}

A six-factor measurement model (self-efficacy, goal progress, environmental support, academic satisfaction, positive affect, and life satisfaction) produced good fit to the data in the full sample: $\mathrm{CFI}=.99, \mathrm{RMSEA}=.023, \mathrm{SRMR}=.026, \mathrm{~S}-\mathrm{B}$ scaled $\chi^{2}(89, \mathrm{~N}=666)=$ 118.97, $p<.001$. The structural model also yielded adequate fit, CFI $=.99$, RMSEA $=.025$, SRMR $=.030, \mathrm{~S}-\mathrm{B}$ scaled $\chi^{2}(92, N=$ $666)=129.10, p<.001$. Although the structural model did not fit the data as well as the measurement model using the $\Delta S-B \chi^{2}$ criterion $\left(\Delta \mathrm{S}-\mathrm{B} \chi^{2}[3]=11.02, p<.05\right)$, the difference in fit between the two models was small in a practical sense $(\Delta \mathrm{CFI}=.00)$, suggesting that the structural model offered an adequate explanation of the relations among the factors.

As shown in Fig. 2, support was found for all but two of the 12 hypothesized paths. In particular, the only non-significant coefficients were for the direct paths from self-efficacy to academic satisfaction and from goal progress to life satisfaction. These non-significant paths suggest that self-efficacy was linked to academic satisfaction indirectly through goal progress and that goal progress was, likewise, linked to life satisfaction indirectly through academic satisfaction. On balance, academic and life satisfaction were each well-predicted in the model $\left(R^{2}=.69\right.$ and .42 , respectively). The $R^{2}$ values for self-efficacy, goal progress, and environmental support were, respectively, .40, .42, and .22. Students were more likely to express satisfaction with their academic lives to the extent that they perceived support for their academic behavior, felt that they were making good progress at their academic goals, and held higher levels of general positive affect. Overall life satisfaction was associated with higher academic satisfaction and positive affect. The paths predicting self-efficacy, goal progress, and environmental support were consistent with hypotheses. 


\subsection{Multiple-group analyses}

We next tested the tenability of the well-being model separately by country and gender and also examined model invariance across country and gender with multiple-groups analyses. To assess invariance of model fit, we first constrained indicator-factor loadings to be equal across each grouping variable (e.g., country) and compared the fit of this constrained model with a model in which the factor loadings were allowed to vary by group. Where the two measurement models (constrained and unconstrained) did not yield substantially different fit, we assumed that the constructs were being measured comparably across groups. We then assessed the invariance of the structural paths across groups. This was done by comparing the fit of a model in which the values of both the factor loadings and the structural paths were constrained to equality across groups against a model in which the factor loadings were constrained to equivalence but the structural paths were allowed to vary by group. Results of all of the multiple-group analyses are presented in Table 2.

\subsubsection{Model fit by country}

The structural model testing the hypothesized paths among constructs by country achieved adequate fit to the data separately in each sub-sample; for the Angola sample, CFI $=.98$, RMSEA $=.032$, SRMR $=.043$, S-B scaled $\chi^{2}(92, n=241)=115.27$, $p<.001$; for the Mozambique sample, CFI $=.99$, RMSEA $=.024$, SRMR $=.034$, S-B scaled $\chi^{2}(92, n=425)=114.22, p<.001$. The SCCT model accounted for relatively large amounts of predictive variance in academic and life satisfaction in both the Angola and Mozambique sub-samples (for academic satisfaction, $R^{2}=.70$, .68, respectively; for life satisfaction, $R^{2}=.43$, .44). The multisample analysis revealed that the measurement and structural models were each invariant across the two sub-samples. In particular, the constrained measurement model did not differ from the unconstrained measurement model on either fit difference criterion. Likewise, the constrained structural model did not differ from the unconstrained structural model on either criterion. Thus, the constructs appear to retain an equivalent latent structure, and the relations among the constructs are similar, across the two national contexts.

\subsubsection{Model fit by gender}

The structural model also achieved adequate fit to the data by gender; for women, CFI $=.99, \mathrm{RMSEA}=.017$, SRMR $=.039$, S-B scaled $\chi^{2}(92, N=250)=98.63, p<.001$; for men, CFI $=.99$, RMSEA $=.024$, SRMR $=.035$, S-B scaled $\chi^{2}(92, N=$ $416)=114.21, p<.001$. The analyses accounted for large amounts of the predictive variance in academic and life satisfaction in both genders (for academic satisfaction, $R^{2}=.63$ in women and .73 in men; for life satisfaction, $R^{2}=.43$ in women and .44 in men). The multisample analysis indicated that the constrained measurement model did not differ in fit from the unconstrained model on either the statistical $\left(\Delta \mathrm{S}-\mathrm{B} \chi^{2}\right)$ or practical criteria $(\Delta \mathrm{CFI})$, suggesting measurement equivalence across gender. Likewise, the structural model with constraints on all paths did not differ in fit from the unconstrained structural model on either criterion; the structural model may thus be viewed as invariant across gender.

\section{Discussion}

The present study sought to test an abbreviated version of the social cognitive work well-being model in a non-Western cultural context, specifically, two African countries, Angola and Mozambique. In addition to extending the model to this cultural context, the study examined how well the social cognitive model fit the data both across countries and gender. The findings suggested that the model fit the data well overall and accounted for sizeable portions of the variance in the two indicators of well-being, academic domain satisfaction and general life satisfaction, in college samples from both countries and among male and female participants.

These findings are largely consistent with those of prior studies of college students conducted in the U.S. (e.g., Hui et al., 2013; Lent et al., 2005) and Portugal (Lent et al., 2009, 2012) indicating that the social cognitive model may shed useful light on social,

Table 2

Fit indices for the multiple-group analyses.

\begin{tabular}{|c|c|c|c|c|c|c|c|c|c|}
\hline Model & $\chi^{2}$ & $\mathrm{~S}-\mathrm{B} \chi^{2}$ & $d f$ & $\Delta \mathrm{S}-\mathrm{B} \chi^{2}$ & $\Delta d f$ & RMSEA $^{a}$ & $\mathrm{CFI}^{\mathrm{a}}$ & $\Delta \mathrm{CFI}^{\mathrm{a}}$ & SRMR \\
\hline \multicolumn{10}{|l|}{ Grouping variable: gender } \\
\hline Measurement model without constraints & 219.64 & 202.11 & 178 & - & - & .014 & .99 & - & .033 \\
\hline Measurement model with constraints on all loadings & 227.13 & 209.40 & 188 & 7.15 & 10 & .013 & .99 & .00 & .035 \\
\hline Structural models without constraints & 237.65 & 219.94 & 194 & - & - & .014 & .99 & - & .038 \\
\hline Structural model with constraints on all structural paths & 246.87 & 228.81 & 206 & 8.75 & 12 & .013 & .99 & .00 & .046 \\
\hline \multicolumn{10}{|l|}{ Grouping variable: country } \\
\hline Measurement model without constraints & 236.43 & $218.68^{*}$ & 178 & - & - & .019 & .99 & - & .035 \\
\hline Measurement model with constraints on all loadings & 246.25 & $228.07^{*}$ & 188 & 9.32 & 10 & .018 & .99 & .00 & .039 \\
\hline Structural models without constraints & 258.01 & $239.80^{*}$ & 194 & - & - & .019 & .99 & - & .042 \\
\hline Structural model with constraints on all structural paths & 268.08 & $249.55^{*}$ & 206 & 9.62 & 12 & .018 & .99 & .00 & .050 \\
\hline
\end{tabular}

\footnotetext{
a Derived from robust maximum likelihood estimation.

$* p<.05$.
} 
cognitive, behavioral, and personality contributors to domain and life satisfaction. Our results also replicated with African students previous findings showing that model fit was invariant across gender in a sample of Mexican American college students (Ojeda et al., 2011). This suggests that the same variables may be useful in accounting for both women's and men's academic and life satisfaction in at least two different cultural contexts.

It should also be noted that the average age of our participants was a bit older (by 4-8 years) than that of the college student samples employed in much of the prior research on the social cognitive well-being model. The age difference may be due to the fact that undergraduates in Angola and Mozambique often enter the workforce after completing secondary education and before enrolling in college. The present findings, therefore, suggest that the well-being model is applicable to students who have somewhat greater work and life experience than is often typical of first and second-year college students in the US and Portugal.

Positive affectivity has been a fairly reliable predictor of students' academic and life satisfaction in this literature. We likewise found that African students with a general tendency to experience positive affect were more likely to feel satisfied with the academic parts of their lives and with their lives as a whole. Despite the knowledge value of such findings, they may offer limited implications for promoting well-being when affective tendencies are viewed in narrowly genetic terms (cf. Lent, 2004). Positive affect can also, however, be viewed in more dynamic, partly modifiable terms (e.g., Watson, 2002). Indeed, some findings suggest that self-efficacy and social support can promote the experience of positive affect over time (e.g., Lent et al., 2009, 2012), and other means of facilitating positive affect (e.g., exercise) have also been discussed in the literature (Watson, 2002).

Of additional potential relevance to practice was the observation that other variables also contributed to prediction of the well-being indicators in our study. In particular, satisfaction in the academic domain was predicted by a combination of academic self-efficacy, goal progress, and environmental supports. Students reported being more satisfied with their academic lives to the extent that they perceived they were making progress at their academic goals and that they had adequate social supports for their academic efforts. It was noteworthy that self-efficacy did not predict domain satisfaction directly in our study; rather its path to domain satisfaction was mediated by goal progress. Prior studies have typically found both direct and indirect relations of self-efficacy to academic satisfaction (e.g., Hui et al., 2013; Lent et al., 2005; Ojeda et al., 2011). The indirect pathway in our study suggests that self-efficacious students are more likely to see themselves as succeeding at their academic goals which, in turn, contributes to their academic domain satisfaction. Interestingly, social support produced the single largest path coefficient to domain satisfaction, which may reflect the importance of social relations to well-being within a collectivist cultural context (Lent, 2004).

Also of potential practical import was the observation that academic satisfaction augmented positive affectivity as a predictor of students' overall life satisfaction. That is, students tended to report greater life satisfaction to the extent that they were satisfied within the academic domain, which likely reflects the centrality of the academic experience to the lives of most college students (Lent et al., 2005). Thus, promoting academic satisfaction (e.g., via goal progress and social support provision) offers a viable route to promoting life satisfaction. Contrary to hypotheses, goal progress did not produce a direct link to life satisfaction; its relation to life satisfaction was rather mediated by academic satisfaction, a finding that has been observed in some other tests of the social cognitive model (Lent et al., 2005).

Although the current findings offer provisional support for the social cognitive well-being model in an African cultural context, several caveats should be noted. First, caution should be exercised in generalizing these findings to other African countries and cultures. The African continent includes a large number of countries, cultures, and languages. Angola and Mozambique share some common features (e.g., Portuguese language by virtue of their colonial histories), and our findings suggested that the model-data fit was invariant across samples from these two countries. However, the relevance and adequacy of the social cognitive model in other African cultural and linguistic contexts remain to be demonstrated. Second, our cross-sectional design precludes inferences regarding causality or temporal precedence among predictors and dependent variables. Third, our study focused on the developmental context of college and young adulthood. Thus, it is not clear to what extent similar social cognitive predictors would help to account for satisfaction in other life domains (e.g., social, family, work) and in other age groups in African countries.

These limitations suggest several directions for future research. In particular, it would be useful to explore the utility of the social cognitive model in other African countries and cultures, as well as in other non-Western cultural contexts, such as in Asia (Sheu, Chen, Lin, \& Chong, 2011). Such cultural extensions need to be accompanied by efforts to assess the measurement equivalence of the social cognitive and well-being measures across diverse samples (cf. Sheu \& Lent, 2009). In addition, cross-sectional methods need to be supplemented by longitudinal and experimental methods that are better suited to examining the model's bidirectional, mediational, and causal assumptions. Other directions for future research include greater study of the model in the context of adult career development (e.g., Badri et al., 2013; Duffy \& Lent, 2009) as well as in pre-college students. Finally, it would be helpful to examine more closely the different methods of operationalizing the social cognitive variables. For example, goal progress has been assessed with both participant-generated, idiographic methods (e.g., Lent et al., 2005, Study 1) and with experimenter-constructed, nomothetic measures (e.g., Lent et al., 2005, Study 2). Further research is needed to document the relative utility of each method in terms of model fit and explanation of variance in well-being outcomes.

\section{References}

Badri, M. A., Mohaidat, J., Ferrandino, V., \& Mourad, T. E. (2013). The social cognitive model of job satisfaction among teachers: Testing and validation. International Journal of Educational Research, 57, 12-24. 
Bandura, A. (1997). Self-efficacy: The exercise of control. New York: W.H. Freeman.

Bentler, P. M., \& Wu, E. J. C. (2005). Eqs 6.1 for Windows user's guide. Encino, CA: Multivariate Software.

Cheung, G. W., \& Rensvold, R. B. (2002). Evaluating goodness-of-fit indexes for testing measurement invariance. Structural Equation Modeling: A Multidisciplinary Journal, 9, 233-255.

Diener, E., Emmons, R. A., Larsen, R. J., \& Griffin, S. (1985). The satisfaction with life scale. Journal of Personality Assessment, $49,71-75$.

Duffy, R. D., \& Lent, R. W. (2009). Test of a social cognitive model of work satisfaction in teachers. Journal of Vocational Behavior, 75, $212-223$.

Feldt, R. C. (2012). Social cognitive model of college satisfaction: A test of measurement and path models. College Student Journal, 46 , $812-822$.

Hu, L., \& Bentler, P. M. (1999). Cutoff criteria for fit indexes in covariance structure analysis: Conventional criteria versus new alternatives. Structural Equation Modeling, 6, 1-55.

Hui, K., Lent, R. W., \& Miller, M. J. (2013). Social cognitive and cultural orientation predictors of well-being in Asian American college students. Journal of Career Assessment, 21, 587-598.

Lent, R. W. (2004). Toward a unifying theoretical and practical perspective on well-being and psychosocial adjustment. Journal of Counseling Psychology, 51, $482-509$.

Lent, R. W., \& Brown, S. D. (2006). Integrating person and situation perspectives on work satisfaction: A social-cognitive view. Journal of Vocational Behavior, 69, $236-247$.

Lent, R. W., \& Brown, S. D. (2008). Social cognitive career theory and subjective well-being in the context of work. Journal of Career Assessment, $16,6-21$.

Lent, R. W., Brown, S. D., \& Hackett, G. (1994). Toward a unifying social cognitive theory of career and academic interest, choice, and performance [monograph]. Journal of Vocational Behavior, 45, 79-122.

Lent, R. W., Nota, L., Soresi, S., Ginevra, M. C., Duffy, R. D., \& Brown, S. D. (2011). Predicting the job and life satisfaction of Italian teachers: Test of a social cognitive model. Journal of Vocational Behavior, 79, 91-97.

Lent, R. W., Singley, D., Sheu, H., Gainor, K., Brenner, B. R., Treistman, D., et al. (2005). Social cognitive predictors of domain and life satisfaction: Exploring the theoretical precursors of subjective well-being. Journal of Counseling Psychology, 52, 429-442.

Lent, R. W., Singley, D., Sheu, H., Schmidt, J. A., \& Schmidt, L. C. (2007). Relation of social-cognitive factors to academic satisfaction in engineering students. Journal of Career Assessment, 15, 87-97.

Lent, R. W., Taveira, M., \& Lobo, C. (2012). Two tests of the social cognitive model of well-being in Portuguese college students. Journal of Vocational Behavior, 80, 362-371.

Lent, R. W., Taveira, M., Sheu, H., \& Singley, D. (2009). Social cognitive predictors of academic adjustment and life satisfaction in Portuguese college students: A longitudinal analysis. Journal of Vocational Behavior, 74, 190-198.

Ojeda, L., Flores, L. Y., \& Navarro, R. L. (2011). Social cognitive predictors of Mexican American college students' academic and life satisfaction. Journal of Counseling Psychology, 58, 61-71.

Satorra, A., \& Bentler, P. M. (2001). A scaled difference chi-square test statistic for moment structure analysis. Psychometrika, 66, $507-514$.

Sheu, H., Chen, H., Lin, W., \& Chong, S. S. (2011, August). Well-being of Taiwanese college students: Testing a social cognitive model. Paper presented at the meeting of the American Psychological Association, Washington, DC.

Sheu, H., \& Lent, R. W. (2009). A social cognitive perspective on well-being in educational and work settings: Cross-cultural considerations. International Journal for Educational and Vocational Guidance, 9, 45-60.

Singley, D., Lent, R. W., \& Sheu, H. (2010). Longitudinal test of a social cognitive model of academic and life satisfaction. Journal of Career Assessment, 18, 133-146.

Verbruggen, M., \& Sels, L. (2010). Social-cognitive factors affecting clients' career and life satisfaction after counseling. Journal of Career Assessment, 18, 3-15.

Watson, D. (2002). Positive affectivity: The disposition to experience pleasurable emotional states. In C. R. Snyder, \& S. J. Lopez (Eds.), Handbook of positive psychology (pp. 106-119). New York: Oxford University Press.

Watson, D., Clark, L. A., \& Tellegen, A. (1988). Development and validation of brief measures of positive and negative affect: The PANAS scales. Journal of Personality and Social Psychology, 54, 1063-1070. 